PROCEEDINGS OF THE

AMERICAN MATHEMATICAL SOCIETY

Volume 131, Number 8, Pages 2305-2311

S 0002-9939(02)06774-6

Article electronically published on November 14, 2002

\title{
A COMPLEX FOR RIGHT-ANGLED COXETER GROUPS
}

\author{
CARL DROMS
}

(Communicated by Stephen D. Smith)

\begin{abstract}
We associate to each right-angled Coxeter group a 2-dimensional complex. Using this complex, we show that if the presentation graph of the group is planar, then the group has a subgroup of finite index which is a 3 -manifold group (that is, the group is virtually a 3-manifold group). We also give an example of a right-angled Coxeter group which is not virtually a 3-manifold group.
\end{abstract}

\section{INTRODUCTION}

Let $\Gamma$ be a finite graph with vertex set $V=\left\{v_{1}, v_{2}, \ldots, v_{n}\right\}$ and edge set $E$. We will define two groups, $A \Gamma$ and $C \Gamma$, and a two-dimensional $\mathrm{CW}$-complex $K \Gamma$.

The group $A \Gamma$ has the presentation

$$
\left.\left\langle v_{1}, v_{2}, \ldots, v_{n}\right| v_{i} v_{j}=v_{j} v_{i} \text { (if } v_{i} \text { and } v_{j} \text { are joined by an edge of } \Gamma \text { ) }\right\rangle .
$$

The group $A \Gamma$ is sometimes referred to as a right-angled Artin group, or graph group.

The group $C \Gamma$ is the quotient $A \Gamma / N$, where $N$ is the normal closure of the set $\left\{v_{1}^{2}, \ldots, v_{n}^{2}\right\}$ in $A \Gamma . C \Gamma$ is called a right-angled Coxeter group, and $\Gamma$ is called its presentation graph. Note that this differs from the usual notion of the presentation graph of a Coxeter group, in that we include edges labeled "2", and exclude the ones labeled " $\infty$ ".

Next we describe the complex $K \Gamma$. The 1-skeleton of $K \Gamma$ is the same as that of the unit cube $[-1,1]^{n}$ in $\mathbb{R}^{n}$ : that is, the set of all points $\left(x_{1}, x_{2}, x_{3}, \ldots, x_{n}\right) \in$ $[-1,1]^{n}$ where $\left|x_{i}\right|<1$ for at most one index $i . K \Gamma$ has $2^{n-2} 2$-cells for each edge of $\Gamma$ : if $v_{i}$ and $v_{j}$ are the endpoints of an edge, then the points $\left(x_{1}, x_{2}, \ldots, x_{n}\right)$ with $\left|x_{i}\right|<1,\left|x_{j}\right|<1$ and $\left|x_{k}\right|=1$ for all $k \neq i, j$ are the interior points of these 2-cells. Note that $K \Gamma$ has $2^{n}$ vertices, $n 2^{n-1}$ edges and $e 2^{n-2}$ faces, where $e$ is the number of edges of $\Gamma$.

It is obvious that $K \Gamma$ is orientable for any graph $\Gamma$, and that if $\Gamma_{1}$ is a subgraph of $\Gamma$, then $K \Gamma_{1}$ is a subcomplex of $K \Gamma$.

Received by the editors October 31, 2001 and, in revised form, March 10, 2002

2000 Mathematics Subject Classification. Primary 20F55; Secondary 05C25, 20F65, 57M20.

Key words and phrases. Right-angled Coxeter group, two-dimensional complex, three-manifold group.

(C)2002 American Mathematical Society 
In [1, it was shown that $\pi_{1}(K \Gamma)$ is a subgroup of the commutator subgroup of $A \Gamma$. Here, we show that it is the full commutator subgroup of $C \Gamma$ :

Proposition 1.1. $\pi_{1}(K \Gamma)$ is isomorphic to $C \Gamma^{\prime}$, the commutator subgroup of $C \Gamma$.

Proof. Let $w$ be a word in the letters $v_{1}, v_{2}, \ldots, v_{n}$. We associate to $w$ a path $P_{w}$ in the complex $K \Gamma$ beginning at the point $p_{0}=(-1,-1, \ldots,-1)$ as follows: if $w$ is the empty word, then $P_{w}$ is the constant path based at $p_{0}$.

Now let $w^{\prime}$ be a word, and let $w=w^{\prime} v_{j}$. Then $P_{w}$ first traces out the path $P_{w^{\prime}}$, and then traverses the edge joining the endpoint $\left(x_{1}, x_{2}, \ldots, x_{n}\right)$ of $P_{w^{\prime}}$ to the point $\left(x_{1}, \ldots, x_{j-1},-x_{j}, x_{j+1}, \ldots, x_{n}\right)$.

It is clear that $P_{w}$ is a loop if and only if $w$ has an even number of occurrences of each letter $v_{i}$, which is the case if and only if the group element represented by $w$ belongs to $C \Gamma^{\prime}$, and that if $w$ and $u$ are two loops based at $p_{0}$, then the path $P_{w u}$ consists of a traversal of $P_{w}$, followed by a traversal of $P_{u}$.

Now, any path in $K \Gamma$ is homotopic to $P_{w}$ for some word $w$. Suppose $P_{w}$ and $P_{u}$ are homotopic paths. Then $w$ can be transformed into $u$ by a finite sequence of (i) insertions and deletions of subwords $v_{i} v_{i}$, and (ii) replacements of subwords $v_{i} v_{j}$ with $v_{j} v_{i}$, where the vertices $v_{i}$ and $v_{j}$ are joined by an edge of $\Gamma$. But this is equivalent to saying that $w$ and $u$ represent the same element of $C \Gamma^{\prime}$.

\section{GRAPHS}

As we are using graphs as a means of encoding group presentations and of describing complexes, we shall need a few preliminary definitions and facts about them. The graphs in this paper will all be finite, with no loops or multiple edges.

Let $n$ be a positive integer. Then $D_{n}$ is the graph with $n$ vertices and no edges (a so-called discrete graph). $C_{n}(n \geq 3)$ is the $n$-cycle; that is, the vertices and edges of an $n$-gon. $K_{n}(n \geq 1)$ is the complete graph with $n$ vertices. Finally, if $m$ and $n$ are both $\geq 1$, then $K_{m, n}$ is the complete bipartite graph; it has one set of $m$ vertices and another set of $n$ vertices. Each vertex in the first set is joined by an edge to each vertex in the second, and there are no other edges.

A graph is planar if it can be represented in the plane by a set of points (representing the vertices) and arcs (representing the edges) so that no two arcs share an interior point. The so-called "Kuratowski graphs," $K_{5}$ and $K_{3,3}$, are famously non-planar.

A planar embedding of a planar graph defines a number of regions; these are the connected components of the complement of the graph in the plane. Note that exactly one of these is unbounded. Each region (except the unbounded one) is homeomorphic to a disk, but the boundary of a region need not be homeomorphic to $S^{1}$. We will call the vertices (edges) on the boundary of the unbounded region exterior vertices (edges) and the rest will be called interior vertices (edges). The bounded regions will be called interior regions. Note that this depends on the particular embedding of the graph in the plane.

We will call a graph a triangulated polygon if it can be embedded in the plane so that the boundary of each interior region is isomorphic to $C_{3}$, and the boundary of the unbounded region is isomorphic to $C_{n}$ for some $n \geq 3$. If $\Gamma$ is a triangulated polygon, then the boundary of the exterior region will be called the boundary of $\Gamma$, and denoted $\partial \Gamma$. It is easy to see that every planar graph can be embedded in a triangulated polygon. 


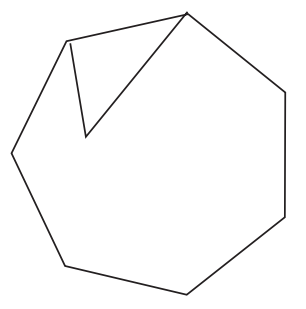

(i)

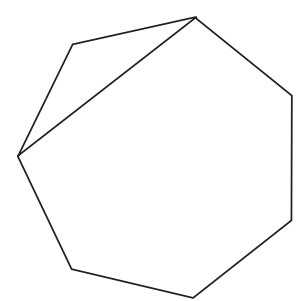

(ii)

FIGURE 1.

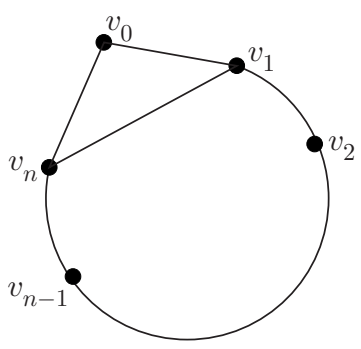

Figure 2. $C_{n}^{+}$

Lemma 2.1. Let $\Pi$ be a triangulated polygon which is not isomorphic to $C_{3}$. Then there is an interior region whose boundary contains either (i) exactly one exterior edge and two exterior vertices, or (ii) exactly two exterior edges and three exterior vertices (see Figure 1 1).

Proof. Let $v_{1}, v_{2}, \ldots, v_{n}$ be the boundary vertices of $\Pi$, reading, say, clockwise around. For each $i$, let $v_{i}^{+}$be the next neighbor of $v_{i}$ in $\Pi$, reading in the clockwise direction from $v_{i+1}$. Since $\Pi$ is a triangulated polygon, there is an edge joining $v_{i}^{+}$ to $v_{i+1}$ for each $i$. If, for some $i, v_{i}^{+}$is not on the boundary of $\Pi$, then $v_{i}, v_{i+1}$ and $v_{i}^{+}$are the vertices of a triangle of type (i) above.

So suppose that each $v_{i}^{+}$lies on the boundary of $\Pi$, say $v_{i}^{+}=v_{k_{i}}$. If $k_{1}=n$, then $v_{1}, v_{2}$ and $v_{1}^{+}=v_{n}$ are the vertices of a triangle, and since $\Pi$ is not a triangle itself, the edge joining $v_{2}$ to $v_{n}$ is an interior edge; that is, the triangle is of type (ii).

If $k_{1}<n$, then either $k_{2}=1$, and there is a triangle of type (ii), as above, or $3 \leq k_{2} \leq k_{1}$. Continuing clockwise around the boundary of $\Pi$, we see that eventually we find $j<k_{1}$ with $k_{j}=j-1$; that is, there is a triangle of type (ii).

\section{The COMPlex $K \Gamma$}

It is clear from the definition that each vertex of $K \Gamma$ has a neighborhood homeomorphic to the cone on $\Gamma$, that is, the space obtained from the direct product $\Gamma \times[0,1]$ by identifying the subset $\Gamma \times\{1\}$ to a single point.

Next, we give some examples of complexes $K \Gamma$.

Definition 3.1. For an integer $n \geq 3$, let $[n]$ denote the number $(n-4) 2^{n-3}+1$. 


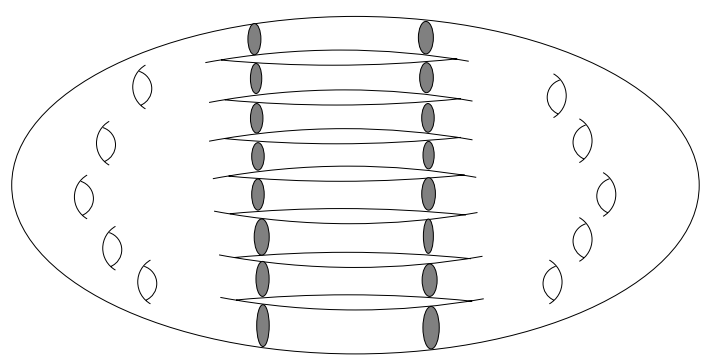

Figure 3. First construction of $K C_{5}^{+}$

Example 3.2. $\quad$ (i) $K D_{n}$ is the 1-skeleton of the $n$-dimensional cube.

(ii) Let $n \geq 3$. Each vertex of $C_{n}$ is incident to two edges. Therefore, in $K C_{n}$, each edge is incident to two faces. Furthermore, the cone on $C_{n}$ is a twodisk. Thus, $K C_{n}$ is a surface. Note that the Euler characteristic of $K C_{n}$ is $2^{n}-n 2^{n-1}+n 2^{n-2}=(4-n) 2^{n-2}$, and so its genus is $(n-4) 2^{n-3}+1=[n]$.

(iii) Let $n \geq 3$. Then by $C_{n}^{+}$we mean the "one-edge union" of $C_{n}$ and $C_{3}$; that is, we attach to $C_{n}$ a new vertex and two new edges, as in Figure 2 We will describe two different constructions of $\mathrm{KC}_{n}^{+}$.

For the first, we think of $C_{n}^{+}$as $C_{n+1}$ with one new edge attached to it. Beginning with a copy of $K C_{n+1}$ (i.e., an $[n+1]$-hole torus), we attach $2^{n-1} 2$-disks to it along their boundaries (see Figure 3 which shows the construction of $K C_{5}^{+}$).

For the second construction, we think of $C_{n}^{+}$as $C_{n}$, with a new vertex and two new edges attached. Let $K_{1}$ and $K_{2}$ be disjoint copies of $K C_{n}$. To $K_{1}$ we attach $2^{n-2}$ closed cylinders (i.e., homeomorphic to 2 -spheres), so that the intersection of each with $K_{1}$ is one of its ends. To this new space we attach $K_{2}$ so that its intersection with each cylinder is the other end of the cylinder (see Figure 4).

Each of the constructions in part (iii) of Example 3.2 leads, under certain hypotheses, to an embedding of $C_{n}^{+}$in a 3-manifold. The first of the following lemmas, corresponding to the first of the constructions, is clear by considering Figure 3 ,

Lemma 3.3. Suppose $K C_{n+1}$ can be embedded in a 3-manifold $M$ in such a way that it bounds an $[n+1]$-hole solid torus in $M$. Then $K C_{n}^{+}$can be embedded in $M$ so that the two copies of $K C_{n}$ bound disjoint $[n]$-hole solid tori.

In the case of the second construction, we have

Lemma 3.4. Suppose $K C_{n}$ can be embedded in a 3-manifold $M$ so that it bounds an $[n]$-hole solid torus in $M$. Then $K C_{n}^{+}$can be embedded in a 3-manifold $\widetilde{M}$ so that the subcomplex $K C_{n+1}$ bounds an $[n+1]$-hole solid torus.

Proof. Let $M_{1}$ and $M_{2}$ be two copies of $M$, each with an embedded $K C_{n}$, and let $T_{1}$ and $T_{2}$ be the solid $[n]$-hole tori bounded by the copies of $K C_{n}$. Form the connected sum $\widetilde{M}=M_{1} \# M_{2}$ by removing a 3-ball from each $T_{i}$, and identifying their boundaries. Now, $\widetilde{M}$ contains the subspace $T=T_{1} \# T_{2}$, whose boundary is the union of the two copies of $K C_{n}$. Thus, one may attach the $2^{n-2}$ cylinders within $T$, to obtain an embedding of $K C_{n}^{+}$in $\widetilde{M}$. 


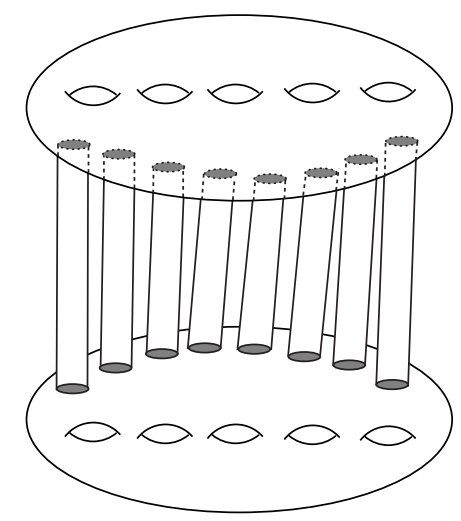

Figure 4. Second construction of $K C_{5}^{+}$

$T$ is homeomorphic to the complement, in $S^{3}$, of two disjoint $[n]$-hole solid tori. It is thus clear from Figure 4 that one component of $\widetilde{M}-K C_{n}^{+}$is homeomorphic to the complement in $S^{3}$ of an [n+1]-hole torus, which is another $[n+1]$-hole torus, and that its boundary is $K C_{n+1}$.

Theorem 3.5. The complex $K \Gamma$ can be embedded in a 3-manifold if and only if $\Gamma$ is a planar graph.

Proof. Suppose $K \Gamma$ is embedded in a 3-manifold $M$; choose a small 3-ball neighborhood of a vertex $v$ of $K \Gamma$. Then it is clear that the intersection of $K \Gamma$ with the boundary $S$ of this 3-ball is homeomorphic to $\Gamma$; the edges of $K \Gamma$ incident with $v$ intersect $S$ in points, and each 2-cell intersects $S$ in an arc joining the points corresponding to the two edges on its boundary which are incident with $v$. Thus, $\Gamma$ is planar.

For the converse, suppose $\Gamma$ is a planar graph. Then $\Gamma$ can be embedded in a triangulated polygon $\widetilde{\Gamma}$, and so $K \Gamma$ is a subcomplex of $K \widetilde{\Gamma}$. Thus, we may assume that $\Gamma$ is a triangulated polygon. If $\Gamma$ is a triangle, the result is obvious, so suppose otherwise; in particular, $\Gamma$ has at least two interior regions. Let $n$ be the number of vertices on the boundary of $\Gamma$. We make the following induction hypothesis: if $\Sigma$ is any triangulated polygon with fewer regions than $\Gamma$, then $K \Sigma$ can be embedded in some 3-manifold $M$ in such a way that each subcomplex of $K \Sigma$ corresponding to the boundary polygon of $\Sigma$ is the boundary of an $[m]$-hole solid torus which is otherwise disjoint from $K \Sigma$, where $m$ is the number of vertices on the boundary of $\Sigma$.

We will show that $K \Gamma$ also possesses such an embedding. By Lemma 2.1 $\Gamma$ has a triangle of type (i) or of type (ii).

Suppose $\Gamma$ has a triangle $t$ of type (i). Let $\Sigma$ be the graph obtained by deleting from $\Gamma$ the exterior edge belonging to $t$. Then $\Sigma$ has fewer regions than $\Gamma$, and its boundary $\partial \Sigma$ is isomorphic to $C_{n+1}$. By induction, $K \Sigma$ can be embedded in a 3-manifold $M$, in such a way that each subcomplex corresponding to $\partial \Sigma$ is the boundary of an $[n+1]$-hole torus which is otherwise disjoint from $K \Sigma$ (note that there are, in all, $2^{N-n-1}$ such tori, where $N$ is the number of vertices of $\Gamma$ ). 
To build $K \Gamma$, we attach 2-cells corresponding to the exterior edge of $t$ to each such $K C_{n+1}$, as in Lemma 3.3. Note that the resulting embedding of $K \Gamma$ satisfies our induction hypothesis.

Now suppose that $\Gamma$ has a triangle $t$ of type (ii). In this case, we let $\Sigma$ be the graph obtained by deleting the two exterior edges of $t$ and their common endpoint. Note that $\Sigma$ has one region fewer than $\Gamma$, and that $\partial \Sigma$ is isomorphic to $C_{n-1}$. Thus, $K \Sigma$ has an embedding in a 3-manifold $M$ as described in the induction hypothesis.

To construct an embedding of $K \Gamma$, we begin with two copies $M_{1}$ and $M_{2}$ of $M$, each with an embedded copy of $K \Sigma$. Let $\phi: M_{1} \rightarrow M_{2}$ be the obvious homeomorphism. For each copy $X$ of $K(\partial \Sigma)$ in $M_{1}$, we remove a 3 -ball from the $[n-1]$-hole torus it bounds. We then do the same for $\phi(X)$, and identify the boundaries of the removed 3-balls. It is easy to see that the resulting space is a 3 -manifold. Finally, we add the cylinders corresponding to the deleted vertex and edges of $t$, as in Lemma 3.4 and it follows that the subcomplexes corresponding to the boundary of $\Gamma$ bound $[n]$-hole tori in this new space.

We recall that if $P$ is a property of groups, then we say that a group $G$ is "virtually $P$ " if some subgroup of finite index in $G$ has property $P$. An immediate consequence of Theorem 3.5 is

Corollary 3.6. If $\Gamma$ is a planar graph, then the group $C \Gamma$ is virtually a 3-manifold group.

Proof. Let $K \Gamma$ be embedded in a 3-manifold $M$. Then $C \Gamma^{\prime}=\pi_{1}(\widetilde{K \Gamma})$, where $\widetilde{K \Gamma}$ is a regular neighborhood of $K \Gamma$ in $M$. Since $C \Gamma^{\prime}$ has finite index in $C \Gamma$, the result follows.

\section{The Kuratowski GRAPHS}

It is well-known that a graph is planar if and only if none of its subgraphs can be contracted onto either $K_{5}$ or $K_{3,3}$. Since $C K_{5}$ is finite, the condition of Corollary 3.6 is not necessary. In this section, we will show that $C K_{3,3}$ is not virtually a 3-manifold group.

Proposition 4.1. Let $H$ be a group, let $\Gamma$ be a graph, and let $G=A \Gamma$. If $H$ has a subgroup isomorphic to $G$, then so has every finite-index subgroup of $H$.

Proof. Let $G$ be generated as a graph group by elements $a, b, c, \ldots$ Then for any $n \geq 1$, it is easy to see that the elements $a^{n}, b, c, \ldots$ generate a subgroup of $G$ which is isomorphic to $G$. It follows by induction that the same is true for $a^{n}, b^{m}, c^{r}, \ldots$ for any choice of integers $n, m, r, \ldots$ all $\geq 1$. But if $H$ has a subgroup which is isomorphic to $G$, and which is generated by elements $a, b, c, \ldots$, then any finiteindex subgroup of $H$ will contain the elements $a^{n}, b^{m}, c^{r}, \ldots$ for some choice of integers $n, m, r, \ldots$, and so will also have a subgroup isomorphic to $G$.

Corollary 4.2. $C K_{3,3}$ is not virtually a 3-manifold group.

Proof. $C K_{3,3}$ is the direct product $H \times H$, where $H=C D_{3}$. Since $K H$ is the one-skeleton of the 3-dimensional cube, its commutator subgroup is free of rank five. Thus, $C K_{3,3}$ contains the direct product of two free groups of rank two, and it is well-known that this group is not a 3-manifold group (see [2] and [3]). But this group is also $A C_{4}$, and so every finite index subgroup of $C K_{3,3}$ has a subgroup isomorphic to it. Thus, no finite-index subgroup of $C K_{3,3}$ is a 3 -manifold group. 


\section{REFERENCES}

1. H. Servatius, C. Droms and B. Servatius, Surface subgroups of graph groups, Proc. Amer. Math. Soc. 106 (1989), 573-578. MR 90f:20052

2. J. Stallings, Coherence of 3-manifold fundamental groups, in Séminaire Bourbaki, Vol. 1975/76, 28 ème année, Exp. No. 481, pp. 167-173. Lecture Notes in Math., Vol. 567, Springer, Berlin, 1977. MR 56:1290

3. G. P. Scott, Finitely generated 3-manifold groups are finitely presented, J. London Math. Soc. 6 (1973), 437-440. MR 52:1660

Department of Mathematics and Statistics, James Madison University, Harrisonburg, VIRGINIA 22807

E-mail address: carl@math.jmu.edu 